Cahiers de recherches médiévales

\title{
Le mythe de Merlin dans la littérature française du $\mathrm{XX}^{\mathrm{e}}$ siècle
}

(Jean Cocteau, René Barjavel, Jacques Roubaud, Théophile Briant, Michel Rio)

Arlette Bouloumié

\section{(2) OpenEdition}

Journals

Édition électronique

URL : https://journals.openedition.org/crm/1833

DOI : $10.4000 / \mathrm{crm} .1833$

ISSN : 1955-2424

Éditeur

Honoré Champion

Édition imprimée

Date de publication : 15 décembre 2004

Pagination : 181-193

ISSN : 1272-9752

Référence électronique

Arlette Bouloumié, «Le mythe de Merlin dans la littérature française du XXe siècle », Cahiers de recherches médiévales [En ligne], 11 | 2004, mis en ligne le 10 octobre 2007, consulté le 15 décembre 2022. URL : http://journals.openedition.org/crm/1833 ; DOI : https://doi.org/10.4000/crm.1833

Ce document a été généré automatiquement le 15 décembre 2022.

Tous droits réservés 


\title{
Le mythe de Merlin dans la littérature française $\mathrm{du} \mathrm{XX}$ siècle
}

(Jean Cocteau, René Barjavel, Jacques Roubaud, Théophile Briant, Michel Rio)

\author{
Arlette Bouloumié
}

1 Merlin est une figure centrale de la mythologie et du folklore celte que nous fait connaître l'œuvre de Robert de Boron ${ }^{1}$ mais surtout celle du Gallois Geoffroy de Monmouth (1100-1155) : La Vita Merlini ainsi que les récits parallèles, l'un irlandais, La Folie Suibhné (en gaélique) ; l'autre écossais, La Vie de Saint Kentigern (XII siècle) ${ }^{2}$.

2 Geoffroy de Monmouth est un érudit gallois latinisant qui fut dans la mouvance des Ducs de Normandie puis des Plantagenêt devenus rois d'Angleterre. Ce fut pendant les dernières années de sa vie qu'il écrivit La Vita Merlini (1148) après Les Prophéties de Merlin et une Histoire des rois de Grande Bretagne (1135-1148) qui sera adaptée en 1155 par Robert Wace sous le titre: Roman de Brut. Il y reprend la légende médiévale galloise de Myrrddhin évoquant un fou exilé dans la forêt et traqué par ses ennemis à la suite d'une bataille qui lui aurait fait perdre la raison.

3 L'analyse du mythe à travers ces différents textes permet de dégager plusieurs composantes du personnage. C'est d'abord un enfant bâtard qui étonne par ses prodiges. Les démons, exaspérés par la descente du Christ dans les enfers et la rédemption des justes, auraient tenté de faire échec à Dieu en produisant une sorte d'Antéchrist, mi-homme mi-démon. Ainsi l'un d'eux aurait-il abusé d'une jeune vierge qui aurait mis au monde un enfant velu, doué d'une perspicacité surnaturelle, Merlin.

4 Un autre aspect du mythe met en valeur le rôle politique de Merlin : barde et prophète, il protège et conseille les rois, Uter et Uterpandragon, il préside à la naissance du roi Arthur et lui conseille la création de la Table ronde qui réunit les chevaliers du roi Arthur, en souvenir de la Cène. Ses prérogatives sont celles des druides. Il a aussi une fonction guerrière de stratège pendant les combats.

5 Merlin est encore l'homme sauvage qui vit en communion avec la nature et qui a des pouvoirs sur elle. Maître des animaux, il apparaît souvent monté sur un cerf ou 
accompagné d'un loup. Il possède le don de métamorphose, surgit et disparaît subitement. Il a des visions, des accès de fureur prophétique vécus comme une sorte de possession. Ces thèmes sont surtout développés dans La Vita Merlini auquel fait écho La Folie Suibhné et La Vie de Saint Kentigern (qui donne un rôle à Lailoken, sorte de Merlin sylvestre ${ }^{3}$ ).

6 La dernière facette du personnage est celle de l'amoureux de Viviane, celui qui, à sa demande, lui enseigne comment procéder pour le garder prisonnier.

De Merlin, le médiéviste Claude Lecouteux écrit :

Son caractère énigmatique derrière lequel se distinguent des traits archaïques résiste à l'analyse et les traditions médiévales, très répétitives, sont embrouillées et témoignent de la littérarisation et de la christianisation d'un individu venu d'ailleurs, d'un lointain autrefois que même les auteurs du XII siècle ne comprenaient sans doute plus 4 .

8 Né de l'union d'une vierge et du Diable, image inversée du Christ né d'une vierge et de Dieu, Merlin est considéré tantôt comme le voyant, le prophète, le poète, le suprême savant, tantôt comme le sorcier, le magicien, l'enchanteur maléfique ${ }^{5}$. Prophète du Graal et de la chevalerie, conseiller du roi Arthur, il est aussi l'enfant prodige, l'homme des bois évoquant une divinité sylvestre ou la victime de l'amour. Ces multiples facettes expliquent la fortune du personnage au XIX siècle dans les littératures allemande, anglaise et française ${ }^{6}$. La littérature française $d u \mathrm{XX}^{\mathrm{e}}$ siècle ne lui fait pas une place moindre comme le montrent la pièce de Cocteau, Les Chevaliers de la Table ronde ${ }^{7}$, créée en 1937, Graal fiction ${ }^{8}$ de Jacques Roubaud publié en 1978, le roman de Barjavel, L'Enchanteur', publié en 1984, et deux œuvres moins connues, celle de Théophile Briant, un poète breton, intitulée Le Testament de Merlin ${ }^{10}$, publiée en 1985 et celle de Michel Rio (lui aussi breton d'origine), Merlin ${ }^{11}$, publiée en 1989. Le rapprochement dans le temps de toutes ces œuvres consacrées à Merlin, témoigne d'un intérêt renouvelé pour le mythe qu'il nous appartient d'éclairer.

Le Merlin de Cocteau retient l'aspect entièrement négatif de l'enchanteur. Cocteau écrit :

L'inspiration n'arrive pas nécessairement de quelque ciel. Il faudrait pour l'expliquer remuer la ténèbre humaine, et sans doute n'en sortirait-il rien de flatteur. Le rôle du poète est humble. Le poète est aux ordres de la nuit. (Ch.T.R. 72)

Il raconte ainsi la genèse de l'œuvre :

En 1934 j'étais malade. Je m'éveillai un matin, déshabitué de dormir et j'assistai d'un bout à l'autre à ce drame dont l'intrigue, l'époque et les personnages m'étaient aussi peu familiers que possible...

C'est trois ans après [...] que j'arrivais à sortir l'ouvrage du vague où je le tenais en marge, comme il nous arrive, malades, le matin, de prolonger nos rêves, de barboter entre chien et loup et d'inventer un monde intermédiaire qui nous évite le choc de la réalité. (Ch.T.R. 72)

L'opium auquel se livre Cocteau après la mort de Radiguet apaise la douleur et favorise le contact avec l'au-delà en supprimant une partie de la barrière entre le conscient et l'inconscient ${ }^{12}$. Mais en même temps il peut stériliser : le moment de la création est le moment intermédiaire, quand la drogue a encore une présence mais laisse place au retour du pouvoir de communication. Merlin entretient donc quelque analogie avec Orphée le poète, mais il représente surtout, pour Cocteau, les enchantements de la drogue avec toute leur ambiguïté. Il donne accès à une illusion de bonheur qui protège de la cruauté du monde. 
Guenièvre dont Ginifer ravale et souille l'image. Merlin participe du démon en ce que, comme lui, il "singe " la création, en donne un double abâtardi. La prononciation déficiente de Ginifer qui dit «es" pour "ex» révèle cette copie imparfaite et grimaçante. Galaad ouvre les yeux à Lancelot. Mais la magie reste interdite à Galaad (Ch.T.R. 141). C'est en utilisant les incantations de Merlin contre lui-même qu'il ramène les chevaliers à Camaalot, le château du roi Artus.

17 L'acte III marque le retour à la réalité pour Artus qui a reçu le message de Merlin où celui-ci dénonce l'adultère de Guenièvre. C'est la vengeance de Merlin. Perdant ses illusions, Artus s'interroge sur les relations de Lancelot et de sa femme. Il tend un piège à Lancelot, découvre la vérité et tue Lancelot :

Vous viviez dans un enchantement mortel lui déclare Galaad. Rien de vrai ne pouvait avoir lieu autour de vous. Maintenant tout est à vif, tout saigne. Plus rien n'enveloppe, n'endort, ne facilite les choses. La vérité commence. Elle est dure. Elle vous fera mal au réveil. (Ch.T.R. 168)

18

incarne donc le monde endormi de l'opium qui permet le rêve éveillé mais qui éloigne du monde réel. Tout refleurit à son départ. Mais la vérité blesse. Artus tue 
Lancelot. L'opium serait-il nécessaire à la vie ? Dans la pénombre du château enchanté d'Artus, le mensonge fait qu'on ne s'y retrouve plus. L'illusion est séduisante comme le diable mais s'oppose au retour de la Grâce incarnée par Galaad. Sans doute le rapport avec le mythe dans cette œuvre de Cocteau est-il lointain, porté par la rêverie. Mais c'est aussi une des interprétations modernes qui renouvellent le plus le mythe de Merlin en lui restituant toute son ambivalence. Jean-Marie Magnan observe :

Le fumeur (Cocteau) s'est fié au mensonge pour s'évader : «Le pavot à la place du Paraclet » n'est-ce pas Merlin déchaînant les maléfices sous le couvert du Graal? Lui-même d'ailleurs ne sait pas toujours à quoi s'en tenir. Une auge de pierre a déposé sur la plage un nouveau chevalier. Sorcellerie ou miracle ? $^{13}$ « grand cerf blanc sorti d'un fourré d'aubépine » $(E, 11)$ qu'il apparait d'abord, lors de sa première rencontre avec Viviane, rappel du "grand cerf branchu » de l'histoire de Grisandole, intégrée à la Suite-Vulgate du Merlin ${ }^{17}$ dans l'édition O. Sommer. L'« arbre bleu » (E, 29), le cèdre, l'accompagne, signe de sa proximité avec le végétal. Il est le maître du vent qu'il déchaîne à volonté sur les ennemis d'Arthur $(E, 19)$. Les expressions « ils accoururent comme l'ouragan » $(E, 19)$ ou « comme la foudre » $(E, 20)$ sont alors à prendre à la lettre. Ainsi se manifeste un lien étroit avec les puissances de la nature ce que symbolise la couleur verte de la robe de Merlin (E, 18 et 31) de sa ceinture $(\mathrm{E}, 31$ et $\mathrm{E}, 166)$ ou de ses yeux $(\mathrm{E}, 31)$. Les feuilles de houx qui le ceinturent $(E, 88)$ ou le coiffent $(E, 18)$ ou ornent sa robe $(E, 31)$ soulignent sa longévité quasi végétale. Les oiseaux font leur nid dans les cheveux de Merlin (E, 85) comme dans un arbre. Les paysans qui le consultent le prennent pour un ancien dieu de la forêt (E, 48 ; E, 178).

23 L'Enchanteur affectionne en effet «l'espluméor », son château d'arbres au cœur de la forêt de Brocéliande où il s'assoit sur son pommier, près de la fontaine de Baranton dont l'eau l'apaise lorsque le diable son père le tourmente. Il participe de «la bienveillance tranquille de la forêt » dont « la force sans limite » l'emplit lorsqu'il tente de fusionner avec elle, se faisant «bois vif, écorce, racine, feuilles vertes et feuilles mortes, graines germées, sèves montantes » $(\mathrm{E}, 69)$ lorsqu'il tente d'oublier Viviane au risque de ne plus retrouver son apparence humaine.

Cahiers de recherches médiévales, 11 | 2004 
Mais parce qu'elle-même est fille de Dyonis, nom qui rappelle « Dionysos, l'ancien dieu des forêts, de la terre et des eaux et du bonheur de vivre en amitié avec les animaux et les arbres ", (E, 57) elle en qui coule aussi le sang de Diane, il ne peut lui échapper.

insiste sur l'aspect sylvestre de Merlin en même temps que sur ses amours avec Viviane. Les exploits guerriers des chevaliers ne sont pas pour autant négligés : Merlin est l'instigateur de la quête du Graal $(\mathrm{E}, 119)$ et de la Table ronde $(\mathrm{E}, 120)$. Il recherche le chevalier assez pur pour mener à bien la quête : il sauve Lancelot en le confiant à Viviane, il éduque Perceval $(E ; 136)$. C'est un héros solaire: «Une lumière chaude rayonnait de lui comme si le soleil l'accompagnait » (E, 166). Merlin incarne les valeurs spirituelles. Il s'oppose aux forces du mal quand il démasque les intentions noires de Morgane, la sœur du roi Arthur (E, 166) et l'alliée de son père le Diable.

La dimension humoristique et parodique n'est pas absente de ce conte : quand Merlin accorde la multiplication de la nourriture comme le Christ accordait celle du pain, il invente ainsi les conserves (E, 181 et E, 279). Il invente de même la flamme de gaz que l'on contrôle par un robinet. Cette actualisation mise au compte de l'esprit malin ne peut que faire sourire.

27 Le caractère protéiforme de Merlin qui surgit sous les apparences les plus diverses contribue encore à l'effet plaisant. Tantôt il prend l'aspect d'un "paysan irlandais coiffé d'un gros bonnet de laine multicolore trempé de pluie, le visage ébouriffé d'une barbe rousse » (E., 215 et 252) au moment pourtant solennel où il songe à construire Stonehenge ; tantôt c'est sous la forme d'un corbeau blanc (E., 244) qu'il se pose sur l'épaule de Lancelot au moment où Arthur hésite à faire chevalier cet inconnu. C'est le moment crucial où le combat entre Dieu et le Diable s'aiguise : «Tu as perdu Arthur pour le Graal, tu as perdu Gauvain, tu as perdu Perceval! Crois-tu que tu vas garder celui-là ? Regarde la reine » (E., 244) ricane le Diable qui annonce ainsi l'adultère de Lancelot et Guenièvre qui rendra ce dernier inapte à la quête du Graal. «Croâ! dit le corbeau » (E., 244). Le corbeau blanc rappelle à la fois la filiation diabolique de Merlin et la colombe du Saint Esprit. Merlin est le héros de ce combat manichéen. Il apparaît enfin sous la forme d'un bûcheron sur une mule avec son fagot (E., 364), traversant un mur couvert d'une tapisserie pour annoncer l'échec de Lancelot dans la quête du Graal.

Mais le roman de Barjavel est avant tout le roman des amours de Viviane et Merlin. C'est sur leur réunion que s'achève le livre, soulignant son orientation optimiste. « Ils vivent depuis ce jour dans la chambre invisible, la chambre d'air, la chambre d'amour, que le temps promène. Elle est là-bas, elle est ailleurs, elle est ici. Un jour elle s'ouvrira comme une graine » (E., 471). Nous sommes loin de la vision désenchantée d'Apollinaire dans L'Enchanteur pourrissant ${ }^{18}$ par exemple et de celle d'Aragon dans le poème «Brocéliande $»^{19}$ qui voit dans Merlin prisonnier d'un arbre enchanté la figure de tous ceux que l'on tient enchaînés (allusion ici à l'occupation allemande).

29 Paradoxalement les satisfactions de la chair sont interdites à Merlin, suscitant ses interrogations. Cette distance prise avec la légende contribue à la légèreté du texte.

Il avait souvent demandé à Dieu de lui expliquer le pourquoi de ce paradoxe dont Viviane et lui - même souffraient tellement: s'il avait fait l'homme et la femme différents et complémentaires, pourquoi était-ce un péché pour eux de se compléter? (...). Pourquoi un homme et une femme qui voulaient s'élever sur le plan spirituel devaient-ils sacrifier le plan sexuel? La joie partagée était-elle condamnable ? La souffrance était-elle le comble de la vertu? (E, 190 )

\section{Il tente de répondre aux interrogations de Viviane :}


Celui qui est appelé à découvrir le Graal doit être vierge. Il faut qu'il soit arraché au fleuve, libéré du désir qui l'entraîne dans le courant (des générations) et fait de lui un esclave indiscernable parmi les milliards de milliards d'esclaves, hommes ou bêtes, accomplissant la même tâche : assurer la continuité de la vie. (E, 352)

De nombreuses pages sont consacrées aux joies de l'amour comme celles qui bouleversent Lancelot et Guenièvre (E, 341). Mais cette nature amoureuse de Lancelot est présentée comme une faiblesse que le diable utilise pour l'empêcher de découvrir le Graal. Lancelot ne fera que donner naissance à celui qui le découvrira (E, 363). L'amour qui imprègne Viviane a imprégné Lancelot, dit Merlin. C'est la forêt qui éduquera Galaad, son fils, lui donnant «les forces essentielles: le besoin absolu de lumière et l'élan vers le ciel.» (E,385). Les écrivains ultérieurs tenteront de résoudre cette contradiction entre l'idéal d'amour et l'idéal mystique de Merlin.

C'est donc une version optimiste de Merlin qu'offre Barjavel: Merlin est jeune, beau, aimant, secourable. C'est un héros solaire, sans ambiguïté, tel qu'un conte de fée peut l'offrir. Le Merlin sylvestre et le héros de l'amour avec Viviane ont autant d'importance dans cette version que l'aspect plus politique de Merlin, à l'origine de la Table ronde. Le ton reste léger, parfois humoristique.

L'humour caractérise aussi le texte de Jacques Roubaud qui se veut une relecture ou une réécriture visant à élucider les mystères des légendes arthuriennes de son enfance, dans l'enchevêtrement des récits médiévaux et de leurs variantes.

Ainsi dans "Conte " par quoi commence Graal fiction, il reprend le récit de la naissance de Merlin mais il suggère malicieusement, dans la confrontation de l'enfant Merlin et du juge chargé de condamner sa mère, que la naissance miraculeuse de Merlin pourrait s'expliquer de façon très naturelle par l'intervention du confesseur Blaise (G.F., 23).

De même, si les relations de Viviane et Merlin font l'objet de versions fort différentes, certaines affirmant que Viviane, en enfermant Merlin, agit sous l'empire de la jalousie à l'égard de Morgane à qui Merlin aurait enseigné la magie noire, d'autres qu'elle voulait préserver sa chasteté, elle, la demoiselle chasseresse, fille de Diane, elles s'expliquent, selon Jacques Roubaud, de façon plus simple et métaphorique :

D'autres, dont je suis, [...], pensent que l'opacité du conte, dans aucune de ses versions, n'est jamais telle qu'elle parvienne à dissimuler tout à fait le lieu, buisson ou grotte, où Merlin s'est vu enserrer et que peut-être il n'a pas tout perdu à sa servitude volontaire. (G.F., 29)

36 Là encore Jacques Roubaud suggère avec humour plus qu'il ne dit ouvertement. Autre mystère: quel est cet "esplumoir» d'où Merlin prophétise? Est-ce une roche inaccessible, toujours verte, dans la montagne, est-ce une tour où «les paroles de Merlin, miel de cette roche ruche se gravaient sur des tablettes de cire » ? (G.F., 32)

Si l'on s'en tient à l'étymologie "l'esplumoir est une cage où un oiseau chanteur est enfermé au moment de la mue : un endroit obscur et chaud où l'oiseau chante dans ses propres plumes. L'esplumoir Merlin est donc l'endroit obscur et chaud au sommet de la roche grifaigne où l'oiseau Merlin chante le futur. On se souvient alors que le «merlin » ${ }^{20}$ est un petit faucon, ce « petit oiseau d'été » que décrit Giraldus Cambrensus dans son Voyage de Galles » (G.F., 33). Ainsi s'expliquerait, selon Jacques Roubaud, que Perceval ait vu une ombre passer et repasser au-dessus de lui tandis qu'une voix parlait pour Merlin, avant que celui-ci ne disparaisse totalement.

38 Jacques Roubaud voit enfin en Merlin l'origine du texte arthurien qui aurait été dicté à Blaise par le prophète enchanteur. «Le livre du Graal n'est autre que le livre de Blaise. 
Il y arrive ce que Blaise entend de Merlin [...]. Les aventures du royaume d'Arthur lui sont à intervalles plus ou moins réguliers racontés par l'enchanteur prophète ». (G.F., 183) Or le Graal lui-même ne serait-il pas un livre, un texte, un Evangile richement orné, s'interroge Jacques Roubaud?

À l'opposé de ces deux versions qui privilégient l'humour et l'aspect homme sauvage apte aux métamorphoses de Merlin (développé dans La Vita Merlini), Le Testament de Merlin de Théophile Briant est un roman pseudo historique. Par ses préoccupations occultistes, il rappelle l'œuvre d'Edouard Schuré qui, dans Les Grandes Légendes de France (1892) évoquait l'initiation d'un Orphée celtique. Il s'inspire également de l'œuvre de Hersart de La Villemarqué qui, dans son œuvre Myrdhin ou l'enchanteur Merlin (1862), tentait de faire le point sur l'histoire de Merlin en qui il retrouvait une figure mythologique héritée de l'antiquité, un héros poétique et en même temps un personnage réel dont les Bretons auraient conservé le souvenir.

Merlin est d'abord présenté comme l'architecte et le maître d'œuvre d'un "bateausorcier» (T.M., 14) qui monte sur la mer à contrevent, l'inventeur de la voile triangulaire. C'est donc un ingénieur mais c'est aussi le poète qui chante «le bardit avant le combat» (T.M., 17) pour mener les guerriers à la victoire, celui dont le seul regard peut clouer au sol l'ennemi (T.M., 18). Sans doute cette force du rhapsode peutelle être attribuée, par ses ennemis, à son commerce avec les dieux infernaux (T.M., 18). Mais c'est avant tout une force spirituelle qui caractérise Merlin, le porte lyre, fondateur de la confrérie où s'incarne l'idéal de la future chevalerie (T.M., 21). «Le Druide » (T.M., 24) est le mot qui le désigne lors de sa première apparition, à la fête du solstice quand les feux des sanctuaires célèbrent la gloire du dieu soleil.

41 La critique est vive à son sujet parmi les guerriers qui ne croient qu'à la force brute et rappellent la vieille querelle sur la nature de l'enchanteur. C'est un " jongleur qui pince la lyre " selon ses détracteurs, c'est un "sorcier qui pactise avec les korrigans" (T.M., 25) selon les autres. Mais ses yeux qui « luisent dans la pénombre comme deux feux Saint-Elme » (T.M., 27) tandis qu'il guide le pilote du navire inspirent la crainte et suggèrent son caractère surnaturel. On le croit de «la race des héros », invulnérable. "Le mage» (T.M., 32) conduit les chevaliers à la bataille de Salisbury qui verra la défaite d'Arthur face aux Saxons et au traître Mordred qui les a ralliés. Merlin connaît cet avenir qu'il ne peut empêcher. Il se compare alors au "convoyeur des ombres " (T.M., 34). Il est le guide qui, pour «le grand voyage», a donné «le viatique de lumière » (T.M., 35).

Merlin a le pouvoir de guérir. Il fait partie des «poètes magiciens dont les mots deviennent des actes.» (T.M., 39). Il est «le dépositaire de la Parole qui relie le terrestre au sacré, et garde sur les puissances rectrices un privilège de contrainte.» (T.M., 42). Pour Théophile Briant, Merlin est donc un grand initié des collèges druidiques. L'auteur le place à l'intersection du druidisme et du christianisme. Ce visionnaire dépositaire des secrets de la religion celte aurait été touché par le message de la nouvelle religion. C'est pour trouver la coupe où Joseph d'Arimathie recueillit le sang du fils de dieu, relique miraculeuse portée jusqu'en Cornouailles, que Merlin aurait fondé l'ordre des Chevaliers de la Table ronde. Avant la grande bataille, il célèbre la communion sous l'espèce du vin, cérémonie qui rappelle la Cène avec le Christ et les prophètes.

43 Merlin s'oppose aux sacrifices humains institués par les druidesses, notamment par Morgane, sœur du roi Arthur et grande prêtresse de l'Ile de Sein. La description les 
rapproche des Bacchantes et leurs menaces préfigurent pour Merlin le sort d'Orphée déchiqueté.

Le patriarche qui a élevé Merlin lui annonce que, s'il est de la race des voyants, doué de seconde vue, il reste « un poète, homme de douleurs » (T.M., 80) qui ne contemple pas " la lumière absolue dans une sérénité sans retour "; il lui reste à parfaire le chemin de son initiation en se retirant dans la solitude des forêts.

Après la bataille de Salisbury où il tue le traître Mordred, Merlin emporte les cadavres d'Arthur et d'Adragante, son disciple, le dernier chevalier de la Table Ronde. Il ravit Adragante au royaume des morts mais, plus heureux qu'Orphée, il le ressuscite.

Le livre II montre les étapes de l'initiation d'Adragante. Merlin apparait désormais "plus grand qu'un héros ou qu'un mage », presque "un surhomme» (T.M., 193). Le dernier et troisième livre évoque une navigation vers l'ouest au cours de laquelle Adragante sauve un naufragé qui s'avère être une jeune fille, Colombe, convertie au christianisme. La tempête les fait s'échouer sur l'île de Sein où une conversation s'engage entre Merlin et la jeune convertie. Celle-ci est sauvée par Merlin de l'immolation à laquelle l'ont condamnée les druidesses de l'île de Sein. Adragante apprend plus tard que Merlin serait mort, lapidé par les druidesses de Morgane qui l'accusent d'avoir trahi la religion celtique. Cette fin l'assimile définitivement à Orphée déchiré par les Bacchantes:

J'étais de la race des poètes comme Orphée de Thrace, mon frère du Sud et parce que j'ai voulu, comme lui, réagir contre les excès sanglants du culte féminin, j'ai dû subir, comme lui, la vengeance de mes ennemies. (T.M., 263)

Mais il se place aussi dans la lignée des grands initiés, de Dionysos déchiré par les Titans, d'Osiris dépecé par Typhon, du Christ crucifié, tous apportant aux hommes un message spirituel (T.M., 263). Il se révèle à Adragante dans son corps glorieux comme un nouveau Christ : sa mission est d'essayer de réconcilier les deux doctrines (l'antique druidisme et le christianisme naissant) sans pour autant renoncer aux croyances traditionnelles des ancêtres :

Je montre par mon exemple qu'on peut devenir fils de Dieu sans proscrire la loi naturelle et que l'humilité suprême réside peut-être dans l'acceptation de ce geste $\mathrm{du}$ sang auquel nous devons notre âme et toutes les promesses attachées à son entité (T.M., 264)

Merlin, «le Druide blanc de Brocéliande » comme il est plusieurs fois désigné dans l'œuvre de Théophile Briant, concilie donc les fonctions de prêtre, de poète, de voyant, de guide. À la fois Orphée et Christ, il est victime de son désir de réconciliation entre la nouvelle et l'ancienne religion. L'amour qui l'unit à Viviane passe ici au second plan et celle-ci garde toute son aura redoutable.

Le Merlin de Michel Rio n'est ni un enchanteur, ni un devin, ni un amant idéal, ni un poète mais un philosophe et un homme d'État. C'est un vieillard qui prend la parole, dans la grotte où il abrite sa retraite depuis cinquante ans, pour faire le bilan de sa vie. "J'ai cent ans [...]. Je porte le deuil d'un monde et de tous ceux qui l'ont peuplé. J'en suis le seul survivant. Dieu lui-même se meurt et Satan ne va guère mieux » (M., 9)

D'emblée s'impose le thème de la mort : la grotte domine en effet la plaine où repose les trois êtres qu'il a le plus aimés : Viviane, Morgane et Arthur. Il s'interroge sur la durée des créations humaines :

J'ai voulu mettre le Diable, dont on dit que je suis issu, au service de Dieu, c'est-àdire de l'homme [...]. Que restera-t-il dans la mémoire des hommes en qui 
cohabitent l'âme et le chaos de ce mélange de Dieu et du Diable? Violence des doux, trahison des fidèles, imprévoyance des sages, lascivité des courtois, adultère et inceste des purs, faiblesse des puissants, idéalité des fins et amoralité des moyens [...]. Restera-il la victoire ébauchée d'une idée ou un ultime échec devant la brutalité des choses? (M., 13)

51 Le récit en flash back se déroule entre deux descriptions de champs de bataille : l'une, en l'année 450 quand Merlin a cinq ans (M., 20), c'est l'effondrement de Rome sous l'assaut des barbares ; l'autre, en l'année 539 quand « le monde venait de s'écrouler une deuxième fois » (M., 143) : c'est la défaite d'Arthur, trahi par Mordred son fils, devant les barbares. La parole du grand-père de Merlin retentit dans sa mémoire, rapprochant ces deux apocalypses identiques : «Il n'y a que la guerre, Merlin » (M., 17, 19, M., 132). Le chaos finit toujours par l'emporter.

Merlin évoque ses origines: il s'est d'emblée perçu comme exclu de la société des hommes et comme voué à la solitude (M., 27). Il est présenté par le confesseur de sa mère comme l'instrument de Dieu pour «faire naître du chaos un homme capable de vaincre le chaos" (M., 28). Le même lui prédit qu'il sera " un sage et un maître entre tous les hommes, un vieillard éternel et un menteur. Je te prédis que ta clairvoyance et le désir du bien t'acculeront à mentir, non à toi-même mais à autrui [...]. Tu n'aimes pas ce monde et tu m'as dit vouloir en inventer un autre. Mais dans toute invention il y a un leurre et la recherche de la vérité même passe par l'illusion. Comment autrement persuader le faible qu'il a des droits, le fort qu'il a des devoirs?» (M., 22). D'emblée le mélange de bien et de mal apparaît comme inéluctable. Merlin est un sage et un marchand d'illusions.

La prédiction s'accomplit. Merlin perd sa mère à neuf ans. «Je me sentais un enfant et un vieillard » (M. 43). «Je fus à l'âge de quinze ans, une sorte de père ayant à aimer un enfant, à éduquer un roi, à inventer un homme.» (M. 57). Il crée les Chevaliers de la Table ronde pour préparer la venue d'un âge d'or, par «la guerre du droit contre la force, de la lumière contre l'obscurité, de l'esprit contre la nature, de Satan contre l'ignorance et de Dieu contre sa propre création » (M. 60) et Merlin atteint presque son but :

Une Table Ronde devenue mythe s'incarnait dans une trinité presque divine: Arthur, Mordred et Lancelot [...]. Cependant dans le ciment vertueux qui soudait cette trinité entraient quelques ingrédients corrosifs jusqu'alors neutralisés [...] par cette obscure générosité de la violence entretenue par le fait de risquer sa vie aux côtés de l'autre et pour l'autre. Ces ingrédients étaient l'inceste, l'adultère et le mensonge. Mais la paix durait et tout tenait ensemble comme si Dieu et Satan s'étaient associés pour porter un même ouvrage à un degré de perfection jamais atteint. Et cet ouvrage dont j'avais jeté les bases était comme le prolongement monumental de ma propre ambiguïté.

Mais la tentative de réconciliation de Dieu et du Diable dans un monde meilleur échoue: le rêve s'effondre quand éclate le scandale de l'amour de Guenièvre et de Lancelot, quand Arthur et Lancelot s'affrontent, que Mordred révèle son origine incestueuse et se révolte contre Arthur.

À l'opposé du Testament de Merlin, le mal incarné par Morgane et Mordred est vu avec une immense compassion par Merlin. Deux scènes se font écho, celle où Morgane enfant demande : «Pourquoi faut-il mourir, Merlin?» $($ M.,65) et celle où Morgane vieillie et aussi effrayante que Méduse meurt dans les bras de Merlin (M., 150).

L'accusation de Morgane contre Dieu exprime la révolte et le désespoir humain : 
Je me scandalise du fait que le centre de tout est sans pensée ni motif, alors que le fruit même du hasard, qui remue faiblement à sa périphérie, parmi d'autres objets errant dans le vide, est, lui, capable de concevoir le but, et que cette capacité ne lui sert qu'à éclairer d'une lumière de plus en plus crue son propre néant. Ainsi je vois bien que Dieu, s'il existe, auteur de tout cela, est mille fois plus cruel et pervers que Satan. (M., 70)

Morgane se voue au mal pour répondre à cette cruauté, parce qu'elle est condamnée au savoir et à la peur. De même Mordred, le fils incestueux d'Arthur et de Morgane est « traître par excès de fidélité, monstre par excès d'idéal, produit parfait du monde que j'avais créé et qu'il avait détruit en raison même de cette perfection »(M., 134).

Merlin construit un monument à son propre échec «parce que la légende construit inlassablement une éternité dont l'histoire s'évertuait à démontrer le mensonge." (M., 148). La fin du livre qui consomme la vanité des efforts humains a un accent camusien. «Je ne veux voir que la mort de l'homme trois fois couché dans le sépulcre et le triomphe de l'été. » (M., 152).

Ainsi chacun fait dire au mythe la vérité qu'il y perçoit: lecture souriante et humoristique de Jacques Roubaud déchiffrant les symboles et nous ramenant à la réalité. Merlin serait le fils naturel d'un abbé qui aurait caché sa faute derrière un mystère théologique, un amant prisonnier et consentant d'une femme paysage devenue grotte et buisson pour le retenir et le combler. Il serait aussi l'auteur mythique de la littérature arthurienne pareil à l'oiseau chanteur qui porte son nom et comme lui intermédiaire entre la terre et le ciel; lecture plus sombre de Cocteau qui s'interroge sur les pouvoirs de l'enchanteur qui prend ici le visage de l'opium. Ou bien Merlin est le détenteur d'une sagesse spirituelle oubliée selon Théophile Briant ${ }^{21}$ ou encore le philosophe désabusé qui réfléchit sur le pouvoir selon Michel Rio ${ }^{22}$.

60 Le mythe le plus propre à exalter le pouvoir de l'homme sur la nature, qu'il soit poète, mage, devin, prêtre ou politique, devient donc souvent l'instrument de la constatation de son impuissance. Si les échos du XIX ${ }^{e}$ siècle retentissent dans le Testament de Merlin de Théophile Briant où Merlin devient le grand initié, Orphée ou Christ, si l'optimisme du conte se retrouve chez Barjavel, le Merlin de Cocteau montre dans les pouvoirs de l'enchanteur l'illusion trompeuse du véritable pouvoir et sa contrefaçon; le Merlin de Michel Rio n'est pas un imposteur mais celui que sa longévité amène à mesurer la vanité des créations humaines. Comme le Merlin de Théophile Briant cherchait à dépasser les antagonismes, religion naturelle des druides et religion du Christ, celui de Michel Rio échoue à unir Dieu et Satan dans une création harmonieuse. Sa compassion pour Morgane, l'instrument du mal, vient de ce qu'il comprend le désespoir métaphysique qui suscite sa révolte. Sa démarche humaniste se heurte au retour inéluctable du chaos et du néant.

61 Au-delà du symbole national du monde celte, Merlin incarne les pouvoirs du créateur et du prophète, poète inspiré le plus souvent, philosophe politique parfois, et ses limites liées à la condition humaine. 


\section{BIBLIOGRAPHIE}

Anne Berthelot, Arthur et la Table ronde, la force d'une légende, Gallimard, coll. « Découvertes », 1996.

Marion Bradley, Les Brumes d'Avalon, Pygmalion, 1986.

Dictionnaire des personnages, Laffont-Bompiani, coll. Bouquins.

Jean Markale, Merlin l'enchanteur ou l'éternelle quête magique, Albin Michel, coll. Espaces libres, 1992.

Joan Woolf, La route d'Avalon.

\section{NOTES}

1.Robert de Boron, auteur d'un Merlin en vers, œuvre perdue mais que l'on connaît par deux textes remaniés, en prose : Le Lancelot-Graal dit couramment La Vulgate (1230), développement des amours avec Viviane et Le Huth Merlin. Voir A. Micha, Étude sur le « Merlin » de Robert de Boron, Genève, éd. Droz, 1980 et La Légende arthurienne, Laffont, Bouquins, 1989, p. 319-430.

2.Textes rassemblés et traduits en français, s.d. Philippe Walter (Grenoble, Ellug, 1999), qui introduit l'ouvrage. Voir aussi, du même auteur, Merlin ou le savoir du monde, Paris, Imago, 2000.

3.Ward, « Lailoken ou Merlin sylvester », Romania 22, 1893.

4.Claude Lecouteux, "Merlin : éléments d'étude stratigraphique », Iris 21, 2001, p. 9.

5.R.L. Wagner, Sorciers et magiciens, Droz, 1939.

6.Simone Bernard Griffiths, Le mythe romantique de Merlin dans l'œuvre d'Edgard Quinet, Champion, 1999. Dean Christopher, A study of Merlin in English Literature from the Middle Ages to the Present Day, Lampeter/Dyfed, 1992.

7.Jean Cocteau (1889-1963), Les Chevaliers de la table ronde, Théâtre, Gallimard, coll. blanche, 1937. Sera abrégé par le sigle. (Ch.T.R.).

8.Jacques Roubaud (1932-), Graal fiction, éd. Gallimard, 1978. Sera abrégé par le sigle G.F. 9.René Barjavel (1911-1985), L’Enchanteur, Denoël, coll. Folio, 1984. Sera abrégé par le sigle (E).

10.Théophile Briant (1891-1956), Le Testament de Merlin, Champion-Slatkine, 1985 (1 ${ }^{\mathrm{e}}$ publication 1975 ). Sera abrégé par le sigle (T.M.).

11.Michel Rio, Merlin, Seuil, coll. Points, 1989. Sera abrégé par le sigle (M).

12. "Cocteau et les mythes » in Revue des Lettres Modernes, 1972, n² 298-303, textes réunis par Jean Jacques Kihm et Michel Décaudin.

13.Jean-Marie Magnan, "Les Chevaliers de la Table ronde, Pièce d'une désintoxication ", in Cahier Jean Cocteau, $n^{\circ}$ 5, Gallimard, 1975, p. 43.

14.Ibid., p. 46.

15.Paul Zumthor, Merlin le prophète, un thème de la littérature polémique, de l'historiographie et des romans, Lausanne, Payot 1943 (Slatkine Reprints, Genève, 1973).

16.Les passages en italique sont soulignés par nous.

17. Cité par Jean-Yves Vadé in « Merlin », Dictionnaire des mythes littéraires, s.d. Pierre Brunel, Le Rocher, 1988, p. 1015. 
18.Guillaume Apollinaire, L'Enchanteur pourrissant, Poésie/Gallimard, 1972, préface de Michel Décaudin. Voir aussi L'Enchanteur pourrissant, Minard, 1972, éd. établie et présentée par Jean Burgos.

19.Louis Aragon, Brocéliande, La Baconnière, 1943, V, « De l'arbre où ce n'est pas Merlin qui est prisonnier » (p. 339-340) :

Le temps torride étreint l'arbre étrangement triste

Tord ses bras végétaux au-dessus de l'étang

Et des chaînes d'oiseaux chargent ce chêne-Christ.

L'enchanteur n'en est plus l'invisible habitant

Et si ce n'est Merlin qui s'est pris à son piège

Qui demeure captif dans le bois palpitant

O Mère c'est en vain lorsque le cœur te fend

Qu'on voudrait te cacher le compte de tes fils

Chacun d'eux dans la terre ou dans l'arbre étouffant

C'est en vain qu'on voudrait te cacher sa torture.

20.Voir l'article de Yves Vadé : « Merlin, l'oiseau et le merlin », Iris, 21, 2000, p. 41-56.

21.Voir également Deepak Choprah, The Return of Merlin (Merlin y apparait comme le détenteur d'une sagesse spirituelle perdue), Arrow Books, 1996, et The Way of the Wizard (série de dialogues entre Merlin et son élève Arthur).

22.Voir également T.H.White, The Book of Merlyn (on y voit l'enchanteur désabusé rejoindre ses amis les animaux pour une mélancolique réflexion sur les risques et les responsabilités du pouvoir).

\section{AUTEUR}

\section{ARLETTE BOULOUMIÉ}

Université d'Angers 